\title{
Relationship Between Serum Vitamin D Levels and Symptoms of Depression in Stroke Patients
}

\author{
Sang-Hyun Kim, MD, PhD, Hyun Seok, MD, PhD, Dong Suk Kim, MD
}

\begin{abstract}
Department of Physical Medicine and Rehabilitation, Soonchunhyang University Bucheon Hospital, Soonchunhyang University College of Medicine, Bucheon, Korea
\end{abstract}

\begin{abstract}
Objective To investigate the correlation between depressive symptoms and serum vitamin D levels in stroke patients.

Methods In total, 126 stroke patients were analyzed. The 25-hydroxyvitamin D (25-OHD) concentration of each patient was used to determine their vitamin D status. Depressive symptoms were assessed using the Beck Depression Inventory II (BDI-II) and the Patient Health Questionnaire 9 (PHQ-9). Functional status was evaluated with the Korean version of the Modified Barthel Index (K-MBI). We compared the clinical questionnaires of a vitamin D-deficient group and a normal group, and evaluated the correlations between BDI-II, PHQ-9, K-MBI, and serum 25-OHD levels.

Results In the vitamin D-deficient group, BDI-II (16.0 \pm 12.1$)$ and PHQ-9 (7.4 \pm 4.2$)$ scores were significantly higher than those of the normal group (BDI-II, 9.1 \pm 7.2 ; PHQ-9, 4.2 $\pm 2.9 ; \mathrm{p}<0.01$ ). In a Spearman correlation analysis, a significant negative correlation was found between serum 25-OHD levels and BDI-II (Spearman $\mathrm{r}=0.177, \mathrm{p}=0.048$ ), but there were no significant correlations between serum 25-OHD levels and PHQ-9 or K-MBI.

Conclusion Vitamin D deficiency was correlated with the symptoms of depression in stroke patients.
\end{abstract}

Keywords 25-hydroxyvitamin D (25-OHD), Stroke, Depression

\section{INTRODUCTION}

Depression is common among stroke patients, and the clinical importance of post-stroke depression is increasing. A recent systematic review indicated that post-stroke

Received April 14, 2015; Accepted August 4, 2015

Corresponding author: Dong Suk Kim

Department of Physical Medicine and Rehabilitation, 170 Jomaru-ro, Wonmi-gu, Bucheon 14584, Korea

Tel: +82-32-621-5114, Fax: +82-32-621-5662, E-mail: nickel0050@gmail. com

(c) This is an open-access article distributed under the terms of the Creative Commons Attribution Non-Commercial License (http://creativecommons. org/licenses/by-nc/4.0) which permits unrestricted noncommercial use, distribution, and reproduction in any medium, provided the original work is properly cited.

Copyright ( 2016 by Korean Academy of Rehabilitation Medicine depression was present in 33\% of stroke survivors [1]. Patients with post-stroke depression show more functional disability, worse rehabilitation outcomes, and increased morbidity and mortality in the first year after a stroke [2]. However, the exact pathophysiology of post-stroke depression remains unknown and several different mechanisms have been suggested $[3,4]$.

Vitamin D deficiency has received increasing attention over the last decade. Lower levels of serum vitamin D have been associated with cardiovascular disease, hypertension, neurodegenerative diseases, diabetes, metabolic syndrome, and cancers [5]. Recently, interest in the potential role of vitamin D in mental health has increased, and it has been suggested that depression is dependent on vitamin D status. This is because vitamin D receptors 
are widely distributed in areas of the human brain that are involved in depression $[6,7]$. However, a direct relationship between vitamin $\mathrm{D}$ deficiency and depression in stroke patients has not been established. Thus, the aims of this study were to compare depressive symptoms between a vitamin D-deficient group and a normal vitamin $\mathrm{D}$ level group, and to investigate the correlation between serum 25-hydroxyvitamin D (25-OHD) levels and the degree of depressive symptoms in stroke patients.

\section{MATERIALS AND METHODS}

All participants were informed of the procedure and objectives of this study. Only patients who provided written, informed consent were selected. Ethical approval was obtained from the Institutional Human Research Ethics Committee (No. SCHBC2013-02-004-001).

\section{Participants}

From May 2014 to November 2014, patients with stroke who were admitted to Soonchunhyang University Bucheon Hospital were recruited for a descriptive correlational study to address the relationship between serum vitamin $\mathrm{D}$ level and symptoms of depression in stroke patients. A total of 126 patients were recruited as in a study by Kwasky and Groh [8], another descriptive correlational study investigating the relationship between serum vitamin D levels and depression in young women. Stroke was confirmed using computed tomography and/or magnetic resonance imaging. The following patients were excluded from the study: those who were diagnosed with any psychiatric illness, including depression, before the stroke; those who had a history of taking antidepressants, antipsychotics, corticosteroids, thyroxine, anticonvulsants, or estrogen; those who had aphasia; and those who could not understand and answer the clinical questionnaires.

\section{Clinical questionnaires}

To measure the patients' depressive symptoms, we used the Beck Depression Inventory II (BDI-II) and Patient Health Questionnaire-9 (PHQ-9). BDI-II and PHQ-9 were developed to detect depression in mental healthcare settings, and have been widely used for detecting poststroke depression $[9,10]$. BDI-II, which consists of 21 queries, was developed to detect depression in mental healthcare settings. Responses are on a scale of 0 to 3 in reference to the past fortnight (total score range 0-63) with higher scores indicating greater severity [11]. A factor analytic study indicated that BDI-II provided a meaningful severity score of depression in stroke patients [9]. PHQ-9 is based on the Diagnostic and Statistical Manual of Mental Disorders, fourth edition (DSM-IV) [12]. Responses are rated 0 to 3 in reference to the past fortnight (total score range 0-27) with higher scores indicating greater severity. The PHQ-9 performs well as a screener for post-stroke depression and discriminates equally well between those with and without post-stroke depression, regardless of age, gender, or ethnicity [10]. Additionally, we evaluated the patients' functional status using the Korean version of the Modified Barthel Index (K-MBI).

\section{Laboratory tests}

Fasting blood samples were collected in 5 -mL tubes within $24 \mathrm{hr}$ of completing the clinical questionnaires, and were centrifuged $\left(3,000 \mathrm{rpm}, 15\right.$ minutes, $\left.4^{\circ} \mathrm{C}\right)$; serum was removed and then frozen at $80^{\circ} \mathrm{C}$ until analysis. Serum 25-OHD was analyzed using isotope-dilution liquid chromatography mass spectrometry (Waters, Milford, MA, USA). Free fraction of serum 25-OHD enters the cerebrospinal fluid through an energy-independent passive mechanism, and a part of the protein bound 25-OHD crosses the brain-blood barrier by active transport $[13,14]$. The concentration of 25-OHD in cerebrospinal fluid (CSF) positively correlates with that in the serum [15]. Although there is no consensus on optimal levels of 25-OHD as measured in serum, vitamin $\mathrm{D}$ deficiency is defined by most experts as a 25-OHD level of less than $20 \mathrm{ng} / \mathrm{mL}$ [5]. The 25-OHD levels were inversely associated with parathyroid hormone levels until they reach $20 \mathrm{ng} / \mathrm{mL}$, but for 25-OHD over $20 \mathrm{ng} / \mathrm{mL}$, parathyroid hormone decreases were not significant [16]. Therefore, the participants were classified into a vitamin D-deficient group $(<20 \mathrm{ng} / \mathrm{mL})$ and a normal group according to the 25-OHD level.

\section{Statistical analysis}

The correlations between the BDI-II, PHQ-9, K-MBI scores and the 25-OHD levels were assessed by Spearman rho coefficient. Demographic data, clinical questionnaires and laboratory tests were compared retrospectively between the vitamin D-deficient group and normal level group using Mann-Whitney U test or $\chi^{2}$ test. 


\section{RESULTS}

In total, 126 stroke patients were analyzed, and $77.8 \%$ of patients were classified into the vitamin D-deficient group ( $<20 \mathrm{ng} / \mathrm{mL}$ ). Baseline characteristics of the patients are shown in Table 1. Mean serum 25-OHD levels in the vitamin $\mathrm{D}$-deficient group and the normal group were $10.8 \pm 4.2 \mathrm{ng} / \mathrm{mL}$ and $28.4 \pm 9.1 \mathrm{ng} / \mathrm{mL}$, respectively. When comparing the BDI-II and PHQ-9 levels, both scores were significantly higher in the vitamin D-deficient group than in the normal group (Table 1).

There was a significant negative correlation between the serum 25-OHD levels and the BDI-II score ( $\mathrm{r}=0.205$, $\mathrm{p}=0.021$; Fig. 1A), but no correlation between serum 25-

Table 1. Demographics and baseline clinical characteristics of stroke patients

\begin{tabular}{|c|c|c|c|}
\hline & Vitamin D deficiency group $(\mathrm{n}=98)$ & Normal level group $(n=28)$ & p-value \\
\hline Age (yr) & $62.1 \pm 14.5$ & $65.0 \pm 9.4$ & 0.217 \\
\hline Sex & & & 0.146 \\
\hline Male & $55(56.1)$ & $20(71.4)$ & \\
\hline Female & $43(43.9)$ & $8(28.6)$ & \\
\hline Time to inclusion (day) & $88.9 \pm 200.2$ & $85.2 \pm 134.5$ & 0.927 \\
\hline Affected hemisphere & & & 0.981 \\
\hline Left & $34(34.7)$ & $10(35.7)$ & \\
\hline Right & $44(44.9)$ & $12(42.9)$ & \\
\hline Others $^{\text {a) }}$ & $20(20.4)$ & $6(21.4)$ & \\
\hline MMSE-K & $24.1 \pm 4.3$ & $23.5 \pm 4.5$ & 0.534 \\
\hline K-MBI & $54.5 \pm 27.8$ & $48.1 \pm 25.9$ & 0.268 \\
\hline BDI-II & $16.0 \pm 12.1$ & $9.1 \pm 7.2$ & $0.004^{*}$ \\
\hline PHQ-9 & $7.4 \pm 4.2$ & $4.2 \pm 2.9$ & $0.000^{*}$ \\
\hline
\end{tabular}

Values are presented as mean \pm standard deviation or number (\%).

MMSE-K, Korean version of Mini-Mental Status Examination; K-MBI, Korean version of Modified Barthel Index; BDIII, Beck Depression Inventory II; PHQ-9, Patient Health Questionnaire-9.

${ }^{\text {a) }}$ Other areas include brain stem, cerebellum, and multiple areas.

${ }^{*} \mathrm{p}<0.01$.

(A)

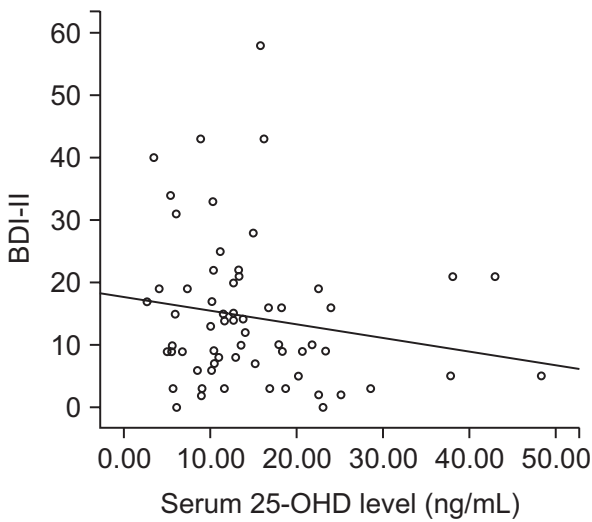

(B)

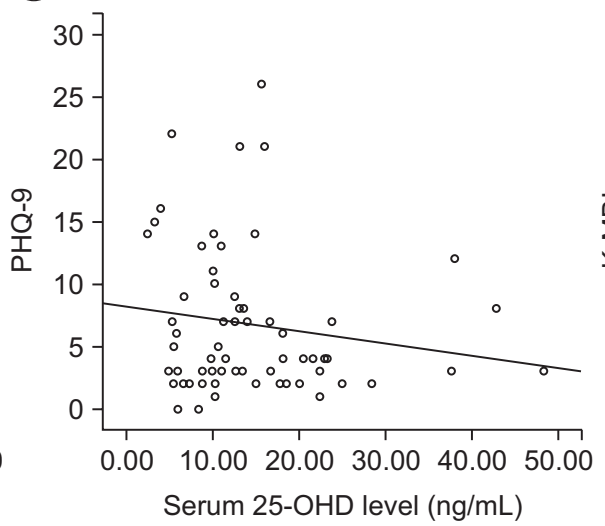

(C)

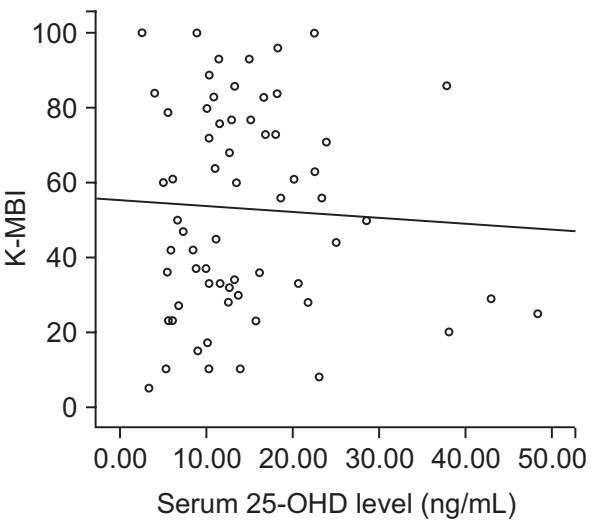

Fig. 1. Scatter plots of correlations between the BDI-II, PHQ-9, and K-MBI scores and serum 25-OHD levels in stroke patients. (A) Correlation between BDI-II and serum 25-OHD levels showed a significant negative correlation (Spearman $r=0.205, p=0.021$ ). (B) Correlation between PHQ-9 and serum 25-OHD levels (Spearman $r=0.156, p=0.081$ ) did not show a significant correlation. (C) Correlation between K-MBI and serum 25OHD levels (Spearman $\mathrm{r}=0.034$, $\mathrm{p}=0.705$ ) did not show a significant correlation. BDI-II, Beck Depression Inventory II; PHQ-9, Patient Health Questionnaire-9; K-MBI, Korean version of the Modified Barthel Index; 25-OHD, 25-hydroxyvitamin D. 
OHD levels and PHQ-9 ( $\mathrm{r}=0.156, \mathrm{p}=0.081$; Fig. 1B) or KMBI scores ( $r=0.034, p=0.705$; Fig. $1 C$ ).

\section{DISCUSSION}

In our study, BDI-II and PHQ-9 scores were significantly higher in the vitamin D-deficient group, showing that depressive symptoms were associated with lower serum 25-OHD levels. A relationship between vitamin D deficiency and depression has been described in several studies. Ganji et al. [17] reported that the prevalence of depression was significantly higher in a population of young adults with vitamin D deficiency. Kjaergaard et al. [18] evaluated vitamin D deficiency and depression in a general population, and established that low serum 25OHD levels were a significant predictor of depression.

The mechanism linking vitamin D deficiency and depression in stroke patients remains unclear, but there are several plausible biochemical mechanisms by which vitamin $\mathrm{D}$ deficiency could change the manifestations of depression in stroke patients. First, depression could be manifested by the inflammatory cytokines associated with vitamin D deficiency. Inflammatory cytokines, such as interleukin-6 (IL-6) and tumor necrosis factor- $\alpha$ (TNF- $\alpha$ ), activate the hypothalamic-pituitary-adrenal axis, resulting in the release of cortisol, which is closely related to manifestations of depression [19]. These inflammatory cytokines can also impair neuroplasticity due to neurotoxicity, which may also be associated with the pathophysiology of depression $[19,20]$. Vitamin D is a potent anti-inflammatory agent and reduces inflammation in the brain [21,22]. Lefebvre d'Hellencourt et al. [23] described the anti-inflammatory activity of vitamin D, and showed a negative correlation between the levels of IL- 6 and TNF- $\alpha$ and vitamin D in microglial cells. Thus, the diminution of the anti-inflammatory effect of vitamin $\mathrm{D}$ in the vitamin $\mathrm{D}$-deficient group might increase depressive symptoms in stroke patients.

Another possible mechanism is through the regulation of neurotransmitters. Vitamin D has been reported to regulate several neurotransmitters. Vitamin D promotes the gene expression of tyrosine hydroxylase, an essential enzyme involved in the synthesis of norepinephrine and dopamine, which are involved in mood regulation and depression [24]. Vitamin D also upregulates serotonin synthesis by activating the transcription of the serotonin- synthesizing enzyme tryptophan hydroxylase 2 in the brain [25]. Norepinephrine, dopamine, and serotonin are associated with the pathophysiology of depression, and these are target neurotransmitters for antidepressants, such as selective serotonin re-uptake inhibitors and serotonin-norepinephrine re-uptake inhibitors [26]. Several lines of experimental evidence have shown that dietary manipulation or direct addition of 25-OHD to the brain alters these neurotransmitter systems. Tekes et al. [27] reported that 25-OHD administered to rodents elevated dopamine and norepinephrine contents in the brainstem, and Smith et al. [28] reported that adult animals treated with high doses of 25-OHD showed increased basal striatal dopamine content. However, we did not evaluate whether the supplementation of vitamin D could improve the symptoms of depression in the stroke patients with vitamin D deficiency. Further studies are required on this.

Several reports have shown that serum 25-OHD levels are negatively correlated with scales of depression [2931]. Polak et al. [30] reported that serum 25-OHD status was significantly negatively associated with a depression scale in young adults. Lee et al. [31] revealed a significant negative correlation between 25-OHD levels and BDI-II scores in their multi-center trial. In this study, a significant inverse correlation was seen between BDI-II scores and serum 25-OHD levels, consistent with the results of previous studies conducted on general populations. And, to our knowledge, this is the first report showing a negative linear correlation between serum 25-OHD levels and symptom scales of depression in stroke patients.

This study has some limitations. First, this study was cross-sectional, so the change in vitamin D levels or progression of depression was not evaluated; thus, we could not determine a temporal or causal association between vitamin $\mathrm{D}$ deficiency and depression in stroke patients. Second, we did not classify our participants by stroke severity or duration after stroke onset. Stroke severity or duration could have an influence on diet or level of activity, which may affect vitamin D metabolism. However, KMBI score and duration after stroke onset did not differ between the two groups, and all of our participants were admitted patients in a university hospital engaged in a conventional rehabilitation treatment program. Thus, daily diet or physical activity would not differ largely between participants and would not have influenced 
our results. Third, the study sample was comprised of admitted patients who were motivated to engage in a rehabilitation treatment program. Thus, these results may not generalize to all stroke patients and the favorable outcome of our study could have resulted, in part, from patient selection bias. Fourth, free fraction of serum 25-OHD enters through an energy-independent passive mechanism, while protein bound 25-OHD is restricted and only a part crosses the brain-blood barrier through active transport $[13,14]$. Therefore, the concentration of serum 25-OHD is higher than those found in human CSF, and serum 25-OHD might not exactly reflect CSF and brain 25-OHD level. However, studies have shown a positive correlation between serum and CSF 25-OHD concentration, and concluded that CSF concentration is determined by the serum concentration, thus measuring serum 25-OHD is reasonable in this study [15,32]. Fifth, there were relatively few study participants $(n=126)$ and they came from a single institution. Further research will be needed considering these limitations.

In conclusion, we showed that BDI-II, PHQ-9 scores differed significantly between a vitamin D-deficient group and a normal group in stroke patients. Additionally, the correlation between serum 25-OHD levels and BDI-II scores was significant. We suggest that vitamin D levels may be a clinical marker for post-stroke depression, and physicians should pay attention to whether stroke patients with vitamin D deficiency have depression. Future studies are needed to confirm our findings and determine whether vitamin $\mathrm{D}$ supplementation could influence post-stroke depression.

\section{CONFLICT OF INTEREST}

No potential conflict of interest relevant to this article was reported.

\section{REFERENCES}

1. Hackett ML, Yapa C, Parag V, Anderson CS. Frequency of depression after stroke: a systematic review of observational studies. Stroke 2005;36:1330-40.

2. Gillen R, Tennen H, McKee TE, Gernert-Dott P, Affleck G. Depressive symptoms and history of depression predict rehabilitation efficiency in stroke patients. Arch Phys Med Rehabil 2001;82:1645-9.
3. Wei N, Yong W, Li X, Zhou Y, Deng M, Zhu H, et al. Post-stroke depression and lesion location: a systematic review. J Neurol 2015;262:81-90.

4. Krishnan V, Nestler EJ. Linking molecules to mood: new insight into the biology of depression. Am J Psychiatry 2010;167:1305-20.

5. Holick MF. Vitamin D deficiency. N Engl J Med 2007; 357:266-81.

6. Berk M, Sanders KM, Pasco JA, Jacka FN, Williams LJ, Hayles AL, et al. Vitamin D deficiency may play a role in depression. Med Hypotheses 2007;69:1316-9.

7. Bertone-Johnson ER. Vitamin D and the occurrence of depression: causal association or circumstantial evidence? Nutr Rev 2009;67:481-92.

8. Kwasky AN, Groh CJ. Vitamin D and depression: is there a relationship in young women? J Am Psychiatr Nurses Assoc 2012;18:236-43.

9. Siegert RJ, Walkey FH, Turner-Stokes L. An examination of the factor structure of the Beck Depression Inventory-II in a neurorehabilitation inpatient sample. J Int Neuropsychol Soc 2009;15:142-7.

10. Williams LS, Brizendine EJ, Plue L, Bakas T, Tu W, Hendrie $\mathrm{H}$, et al. Performance of the PHQ-9 as a screening tool for depression after stroke. Stroke 2005; 36:635-8.

11. Turner A, Hambridge J, White J, Carter G, Clover K, Nelson L, et al. Depression screening in stroke: a comparison of alternative measures with the structured diagnostic interview for the diagnostic and statistical manual of mental disorders, fourth edition (major depressive episode) as criterion standard. Stroke 2012;43:1000-5.

12. Kroenke K, Spitzer RL, Williams JB. The PHQ-9: validity of a brief depression severity measure. J Gen Intern Med 2001;16:606-13.

13. Eyles DW, Burne TH, McGrath JJ. Vitamin D, effects on brain development, adult brain function and the links between low levels of vitamin D and neuropsychiatric disease. Front Neuroendocrinol 2013;34:4764.

14. Pardridge WM, Sakiyama R, Coty WA. Restricted transport of vitamin $\mathrm{D}$ and $\mathrm{A}$ derivatives through the rat blood-brain barrier. J Neurochem 1985;44:1138-41.

15. Balabanova S, Richter HP, Antoniadis G, Homoki J, Kremmer N, Hanle J, et al. 25-Hydroxyvitamin D, 24, 25-dihydroxyvitamin D and 1,25-dihydroxyvitamin 
D in human cerebrospinal fluid. Klin Wochenschr 1984;62:1086-90.

16. Malabanan A, Veronikis IE, Holick MF. Redefining vitamin D insufficiency. Lancet 1998;351:805-6.

17. Ganji V, Milone C, Cody MM, McCarty F, Wang YT. Serum vitamin $\mathrm{D}$ concentrations are related to depression in young adult US population: the Third National Health and Nutrition Examination Survey. Int Arch Med 2010;3:29.

18. Kjaergaard M, Joakimsen R, Jorde R. Low serum 25hydroxyvitamin D levels are associated with depression in an adult Norwegian population. Psychiatry Res 2011;190:221-5.

19. Han QQ, Yu J. Inflammation: a mechanism of depression? Neurosci Bull 2014;30:515-23.

20. Hayley S, Poulter MO, Merali Z, Anisman H. The pathogenesis of clinical depression: stressor- and cytokine-induced alterations of neuroplasticity. Neuroscience 2005;135:659-78.

21. Cekic M, Sayeed I, Stein DG. Combination treatment with progesterone and vitamin D hormone may be more effective than monotherapy for nervous system injury and disease. Front Neuroendocrinol 2009;30:158-72.

22. Kesby JP, Eyles DW, Burne TH, McGrath JJ. The effects of vitamin $\mathrm{D}$ on brain development and adult brain function. Mol Cell Endocrinol 2011;347:121-7.

23. Lefebvre d'Hellencourt C, Montero-Menei CN, Bernard R, Couez D. Vitamin D3 inhibits proinflammatory cytokines and nitric oxide production by the EOC13 microglial cell line. J Neurosci Res 2003;71:575-82.

24. Newmark HL, Newmark J. Vitamin D and Parkinson's disease: a hypothesis. Mov Disord 2007;22:461-8.

25. Patrick RP, Ames BN. Vitamin D hormone regulates serotonin synthesis. Part 1: relevance for autism. FASEB J 2014;28:2398-413.

26. Blier P. Neurotransmitter targeting in the treatment of depression. J Clin Psychiatry 2013;74 Suppl 2:19-24.

27. Tekes K, Gyenge M, Folyovich A, Csaba G. Influence of neonatal vitamin A or vitamin D treatment on the concentration of biogenic amines and their metabolites in the adult rat brain. Horm Metab Res 2009;41:277-80.

28. Smith MP, Fletcher-Turner A, Yurek DM, Cass WA. Calcitriol protection against dopamine loss induced by intracerebroventricular administration of 6-hydroxydopamine. Neurochem Res 2006;31:533-9.

29. Bertone-Johnson ER, Powers SI, Spangler L, Brunner RL, Michael YL, Larson JC, et al. Vitamin D intake from foods and supplements and depressive symptoms in a diverse population of older women. Am J Clin Nutr 2011;94:1104-12.

30. Polak MA, Houghton LA, Reeder AI, Harper MJ, Conner TS. Serum 25-hydroxyvitamin D concentrations and depressive symptoms among young adult men and women. Nutrients 2014;6:4720-30.

31. Lee DM, Tajar A, O'Neill TW, O'Connor DB, Bartfai G, Boonen S, et al. Lower vitamin D levels are associated with depression among community-dwelling European men. J Psychopharmacol 2011;25:1320-8.

32. Holmoy T, Moen SM, Gundersen TA, Holick MF, Fainardi E, Castellazzi M, et al. 25-hydroxyvitamin D in cerebrospinal fluid during relapse and remission of multiple sclerosis. Mult Scler 2009;15:1280-5. 